\title{
Neurological complications in dengue infection: a review for clinical practice
}

\author{
Complicações neurológicas na dengue: uma revisão para a prática clínica \\ Marzia Puccioni-Sohler ${ }^{1,2}$, Carolina Rosadas², Mauro Jorge Cabral-Castro 1,3
}

\begin{abstract}
Dengue is an important global public health problem. The World Health Organization estimates that 2/5 of entire world population are in risk of dengue infection. Almost 50 millions cases occur annually, with at least 20 thousand deaths. The etiological agent of this acute febrile disease is a single-strand positive-sense RNA virus of Flavivirus genus. It is an arboviral disease transmitted by Aedes sp. mosquitoes (Aedes aegypti and $A$. albopictus). Most infected individuals present asymptomatic infection, but some may develop clinical signs. Therefore, a wide spectrum of illness can be observed, ranging from unapparent, mild disease, called dengue fever, to a severe and occasionally fatal dengue hemorrhagic fever/dengue shock syndrome. Currently, neurological manifestations related to dengue infections are increasingly been observed and appears as a challenge for medical practice. In this study the neurological complications of dengue infection will be reviewed, focusing a better understanding of the disease for the clinical practice.
\end{abstract}

Keywords: dengue, nervous system, diagnosis.

\section{RESUMO}

A dengue é um importante problema de saúde pública. A Organização Mundial de Saúde estima que 2/5 da população mundial encontra-se em risco de desenvolver a infecção. Cerca de 50 milhões de casos ocorrem anualmente, com ao menos 20 mil mortes. 0 agente etiológico desta doença febril aguda é um vírus RNA, do gênero Flavivirus. Este arbovírus é transmitido pelo mosquito Aedes sp. A maioria dos indivíduos infectados apresenta infecção assintomática, porém alguns desenvolvem sintomas clínicos. Estes manifestações podem variar desde uma doença inaparente, branda, conhecida como febre da dengue, até uma forma severa, sendo fatal em alguns casos como na febre hemorrágica da dengue/síndrome de choque da dengue. Atualmente, manifestações neurológicas associadas à dengue são cada vez mais frequentes, tornando-se um desafio na rotina médica. Neste estudo, as complicações neurológicas da dengue serão revisadas, com ênfase na melhor compreensão acerca da doença para a prática clínica.

Palavras-Chave: dengue, sistema nervoso, diagnóstico.

The dengue virus belongs to the Flaviviridae family. There are four closely related, but antigenically different virus serotypes: DENV1 to DENV4. An infection caused by one of them can cause dengue fever and, rarely, most severe form of the disease. The infection provides life-long protective immunity to the same subtype, but no immunity against the other three serotypes. Moreover, previous infection with a different subtype increases the chances of developing dengue hemorrhagic fever. This is due to a phenomenon called antibody dependent enhancement ( $\mathrm{ADE}$ ). In $\mathrm{ADE}$, the heterotypic non-neutralizing antibodies form complexes with dengue virus, which infect mononuclear phagocytes with enhanced efficiency. Consequently, a higher number of host cells are infected, improving viral replication, worsening the clinical signs. Thus, this may also contribute to the development of neurological and other complications ${ }^{1}$.

The neurological manifestations are mainly associated with DENV-2 and DENV-3. These serotypes were found in cases of encephalitis, meningitis and myelitis ${ }^{2,3}$. DENV-4 was also detected in brain cells by immunohistochemistry and in cerebrospinal fluid (CSF) of a patient with encephalitis ${ }^{4}$.

The present article highlights some important points that should be addressed when evaluating a possible case of neurological complication of dengue virus infection.

\footnotetext{
1Professor and Chief, Clinical Pathology Service/ Cerebrospinal Fluid Laboratory, Clementino Fraga Filho University Hospital/Federal University of Rio de Janeiro, HUCFF/UFRJ, and Federal University of Rio de Janeiro State, UNIRIO, Rio de Janeiro RJ, Brazil;

${ }^{2}$ Pos-Graduate Student, Program of Infectious Diseases, School of Medicine of the Federal University of Rio de Janeiro, UFRJ, Rio de Janeiro RJ, Brazil; ${ }^{3}$ Pos-Graduate Student, Paulo Goes Microbiological Institute/ Federal University of Rio de Janeiro, UFRJ, Rio de Janeiro RJ, Brazil.

Correspondence: Marzia Puccioni-Sohler; Praia do Flamengo 66 / Bloco B / conj. 219-220;22210-903 Rio de Janeiro RJ - Brazil; E-mail: mpuccioni@hucff.ufrj.br Support: Fundação de Amparo à Pesquisa do Rio de Janeiro (FAPERJ), CAPES.

Conflict of interest: There is no conflict of interest.
} 


\section{Dengue and the nervous system}

The association of dengue infection and neurological abnormalities was first described by Sanguansermsri and colleagues in 1976, in a patient presenting with encephalopathy ${ }^{5}$. Although rare (1-5\% of dengue cases), the neurological involvement have been increasingly reported in dengue epidemics. Furthermore, the cases may be underestimated ${ }^{6}$. The neurological complication can occur in patients with few or no signs of previous dengue infection. The age patients range between few months to 79 years old, being more frequent in children. In most of the cases, the neurological manifestations appear between two and 30 days after the onset of the fever ${ }^{7}$. The neurological symptoms are diverse and related to the location of the lesion. Headache, alteration of consciousness, irritability, insomnia, seizures and focal neurological deficit associated with encephalitis, encephalopathy and stroke pictures are the most common symptoms observed during the acute dengue. Other neurological manifestations, such as motor deficit can occur in acute dengue infection in cases of myelitis and myositis, or during post-dengue stage, in patients with polyradiculoneuritis, encephalomyelitis, neuromyelitis optica, polyneuropathy and mononeuropathy ${ }^{8}$.

The neuropathogenesis of DENV infection is still poorly understood. Viral and host factors may play an important role in the neurological disorders associated with Dengue. In this context, direct viral infection of central nervous system (CNS), autoimmune reaction, metabolic and hemorrhagic disturbances can be involved in the pathogenesis?.

\section{Central nervous system DENV infection}

The Dengue virus infection of the CNS has been confirmed by the detection of viral antigens and DENV RNA in brain tissue and cerebrospinal fluid (CSF) samples. The entry of the virus into the brain seems to occur through infiltrates of infected macrophages ${ }^{10}$.

The relation between viral factors and neuropathogenesis was demonstrated by Bordignon et al. (2007). The authors reported that a mutant DENV-1 caused an extensive leptomeningitis and encephalitis in mice. The hypothesis is that the mutant virus presented neurotropism, crossing the bloodbrain barrier. Other evidence of DENV neurotropism is the detection of intrathecal synthesis of specific antibodies in patients with dengue myelitis ${ }^{11}$.

Encephalitis, myelitis and meningitis are the most important neuroinvasive diseases associated with dengue. However, unlike other viral infections, meningitis due to dengue virus is rare, being more frequent in children. In these cases, the clinical manifestation is similar to other viral meningitis ${ }^{3}$.

Encephalitis is the most common neurological manifestation of dengue infection ${ }^{12}$ and the main symptoms include seizures, altered consciousness, and headaches. Only half of individuals with encephalitis presents the classical symptoms of dengue infection (myalgias, diarrhea, joint or abdominal pain, rash and bleedings). This fact contributes to the underestimation of cases. The diagnosis criteria for dengue encephalitis consists of: fever and acute signs of cerebral involvement associated with the presence of anti-dengue IgM or dengue RNA in the serum and/or CSF added to the exclusion of other causes of viral encephalitis and encephalopathy $^{13}$. In these cases computed tomography (CT) and magnetic resonance imaging (MRI) findings may be normal. Encephalitis secondary to dengue infection usually has a benign outcome. However some patients exhibit persistent neurological symptoms ${ }^{14}$. Glucocorticoid can be used, which highlights the importance of an early diagnosis for a good prognosis ${ }^{15}$.

Myelitis associated with dengue infection is also uncommon (9.5\% to $15 \%)$. It appears 7-30 days following the onset of dengue infection ${ }^{7}$. In these cases neurological symptoms, as paraparesis and sphincter dysfunction, may persist. Magnetic resonance imaging (MRI) usually demonstrates hyper-intense signal at the thoracic spinal level ${ }^{14}$.

\section{Autoimmune reaction in DENV infection}

Autoimmune reaction can occur after DENV infection as a result of the deposition of immune complexes in nervous system. Guillain-Barré (GBS) and Miller Fisher syndrome, neuromyelitis optica and optic neuritis and acute disseminated encephalomyelitis (ADEM) have been reported and related to immunological imbalance in dengue infection ${ }^{15}$.

The pathogenesis of ADEM associated with dengue suggests that the symptoms result from autoimmune reaction causing several areas of white matter brain lesions. Thoracic spinal cord can also be affected, showing demyelinating lesions ${ }^{15}$.

GBS was described in 30\% of the neurological manifestations associated with dengue infection ${ }^{7}$. The clinical presentation of GBS cases induced by dengue is similar to those caused by other infections, with ascending paraparesis as the main manifestation ${ }^{16}$. Treatment is usually effective and the prognosis is good 7 . A case of Miller Fisher associated with dengue was reported presenting spontaneous recover ${ }^{17}$. The clinical manifestations of GBS result from cell-mediated immunological reactions. Dengue virus would initiate the immunological event and myelin or axons could be the target of this immune response ${ }^{18}$. In endemic areas, dengue infection should be investigated in cases of GBS?

Finally, rare cases of fibular, ulnar, long thoracic, and phrenic neuropathy have also been related to dengue infection. Cranial palsy may also occur, mostly with oculomotor and facial nerves involvement ${ }^{9}$.

Benign acute myositis in dengue infection could be consequent of direct viral invasion and/or immune-mediated damage of muscle fibers ${ }^{18}$. In some areas, up to $50 \%$ of benign acute childhood myositis cases occur in dengue infection. 
Myositis is considered a benign illness with a wide clinical spectrum, ranging from mild proximal asymmetrical weakness of the lower limbs to severe, rapidly progressing, limb, trunk, and respiratory muscle weakness. Severe fatal cases are not common. Serum creatine phosphokinase levels are always elevated. Muscle biopsies have revealed histopathological changes (perivascular mononuclear infiltrate, lipid accumulation, foci of myonecrosis, and an increase in mitochondria number) ${ }^{19}$.

\section{DENV and metabolic disturbance}

In acute phase of dengue infection, dengue shock syndrome (DSS), brain edema, cerebral anoxia, metabolic acidosis, electrolyte disturbances, vasculitis, liver and kidney failure can cause encephalopathy ${ }^{20}$.

\section{Hemorrhagic disturbances in DENV infection}

In some cases of acute infection, brain hemorrhage has been reported. It is associated with thrombocytopenia and endothelial damage, with increased vascular permeability and subsequent leakage of fluid.

In both hemorrhagic and metabolic disturbances, pathological studies revealed non-specific lesions, edema, vascular congestion, and focal hemorrhages ${ }^{15}$.

\section{Laboratory diagnosis of dengue infection}

\section{Dengue acute infection}

The gold standard for the diagnosis of dengue infection is still the virus isolation in cell culture followed by identification using fluorescent antibody. However, this technique is not used in clinical routine ${ }^{21}$. In this context, the diagnosis of acute dengue infection is based on the detection of viral antigens, viral RNA or dengue specific antibodies in blood. The antibody detection (IgM and/or IgG) using enzyme-linked immunosorbent assay (ELISA) is the most widely used technique for dengue diagnosis. It has high sensitivity and specificity ${ }^{22}$.

However, some important issues have to be addressed. In a primary infection, for example, the imunnoglobulin $\mathrm{M}$ (IgM) is the first to be synthesized and presents a higher titer. In these cases, serum IgM antibodies can be detected from the 5th to 30-60th days after the onset of symptoms, with $92 \%$ of sensitivity and $99 \%$ of specificity. On the other hand, the IgG appears in low titers at the beginning of symptoms and increases slowly at the end of the first week of the onset of illness. Thus, in recent infection, the detection of dengue IgM antibodies in serum or the increase of dengue IgG titer in paired samples can be used to confirm serological diagnosis. In contrast, in secondary infection, high levels of IgG are detectable even in the acute phase, responding with titers too high in the next two weeks. The kinetics of the response of IgM varies, appearing later during the febrile disease, often preceded by IgG. In some cases, however, IgM is not detected in secondary infections ${ }^{22}$. Specific antibodies may also be detected by complement fixation technique, neutralization test, hemagglutination inhibition assay, or by an immunochromatographic test. The latter is being increasingly used for the rapid diagnosis of dengue infection ${ }^{23}$.

Another point that should be emphasized is the strong cross-reactivity that occurs between members of the Flaviviridae family in indirect immunoassays. The interpretation of serological results can be impaired in cases of others flavivirus infection, such as St. Louis encephalitis, Japanese encephalitis, West Nile fever, and yellow fever. These conditions can also cause similar clinical manifestation ${ }^{24}$.

The detection of non-structural 1 (NS1) viral antigen is used for diagnostic purpose too. This protein is present at the onset of early symptoms and may persist until the fourteenth day after infection, which makes it useful for the early diagnosis of infection. Commercial kits using different methods have been employed for this analysis, such as immunoassays like ELISA test ${ }^{22}$. The commercial methods used for NS1 antigen detection in serum and plasma presented $52 \%$ and $66 \%$ of sensitivity and $90 \%$ and $100 \%$ of specificity ${ }^{23}$. Currently, an immunochromatographic commercial kit that simultaneously detects the presence of IgG, IgM and NS1 antigen is available.

Nucleic acid tests (NATs) as the reverse transcription followed by polymerase chain reaction (RT-PCR) are also used as a direct test. It can amplify and detect the genetic material of dengue virus. It's considered a more sensitive technique than virus isolation in cell culture. The sensitivity can vary from $93 \%$ to $100 \%$, depending on the serotype evaluated. It needs to be performed during viremia. Thus, NATs test can be done early after the infection, before IgM and IgG become detectable. Some varieties of RT-PCR have been developed and presented good efficiency by detecting and differentiating four serotypes of dengue virus using the methodology of semi-nested RT-PCR multiplex. In this technique, a reverse transcription is done, followed by two rounds of PCR, using primers serotypes-specific in the second stage of the technique ${ }^{25}$. Another type of NATs is the real time RT-PCR. This technique is also based on the reverse transcription followed by PCR. Recently, the Center of Disease Control (CDC) from United States developed the first Food and Drug Administration (FDA) approved real time RT-PCR for detection and typing of DENV nucleic acid in suspected cases in the USA ${ }^{26}$. The real time RT-PCR can be also used as a quantitative test in order to determine the viral load. However, this is still an "in house" technique and a rigorous previous validation is necessary before the implementation in clinical routine ${ }^{27}$.

\section{Neurological disease associated with DENV infection: diagnosis}

The presence of dengue IgM, viral antigens or virus RNA in patients with acute neurological symptoms is sufficient for 
the diagnosis of neurological disease associated with dengue virus infection. However, this diagnosis may become difficult due the possibility of neurological manifestation in oligosymptomatic/asymptomatic dengue cases. In this context, blood tests, CSF analysis and MRI images contribute not only to confirm the diagnosis and to exclude other possible differential causes of neurological alteration. These complementary exams help to monitor the patients and to a better understanding of disease pathogenesis. Routine hematologic, biochemical, and liver function, tests for rheumatic diseases, $\mathrm{B} / \mathrm{C}$ hepatitis, and human immunodeficiency virus-1 (HIV-1) should be carried out in all suspected patients. Routine CSF analysis include total and specific cell count, determination of protein and glucose/lactate concentration, a smear and culture for bacteria and fungi, an assessment of blood-CSF barrier function by albumin quotient (CSF/serum), detection of intrathecal synthesis of total IgG, and measurement of specific antibodies against syphilis, cytomegalovirus, EpsteinBarr, and herpes simplex viruses ${ }^{9}$.

\section{Cerebrospinal fluid analysis in suspected patients}

CSF examination contributes to the diagnosis of neurological disorders associated with dengue. However, CSF analysis may be normal. Nonetheless, this does not exclude the diagnosis of neurological manifestations associated with dengue, once up to $50 \%$ of patients with encephalitis may have normal $\mathrm{CSF}^{7}$. Notwithstanding, in those cases, the CSF analysis still is of utmost importance ${ }^{9}$. In this respect, the CSF findings and possible neurological manifestation are resumed in Table 1.

Regarding specific tests for dengue infection in CSF, specific antibody (IgM and IgG), RNA or viral antigen should be also evaluated. Antibodies can be detected in CSF in the early stages of dengue CNS infection up until 5-7 days after the onset of neurological symptoms ${ }^{7,28}$. The dengue IgM detection by ELISA presented a high specificity (97-100\%) but the sensitivity varied between $0 \%$ and $73 \%$, depending on the method used. The absence of specific IgM detection in CSF, however, does not exclude dengue as the causative agent of neurological disorder ${ }^{6,728}$. It is important to note that the detection of specific IgG in CSF is not a useful diagnostic tool, since they may be due to a prior infection and they can cross the blood-CSF barrier ${ }^{9}$. In relation of intrathecal synthesized dengue specific antibodies, previous study demonstrated that this is a potential marker of myelitis associated with dengue infection ${ }^{29}$.

The detection of NS1 antigen in the CSF of dengue patients by ELISA exhibited a sensitivity of $50 \%$ and specificity of $100 \%$. The combined use of these two markers (NS1 Ag and specific IgM) in CSF increases the sensitivity of dengue diagnosis to $92 \%^{28}$.

The detection of dengue viral RNA in CSF, using PCR techniques presented variable results $(0-83 \%)^{2,6,28}$. This discrepancy may be associated to different viral phenotypes which may present distinct neurovirulent and neuroinvasive properties. Other factor that may affect the result includes the stage of the disease, once the viral RNA is detected only in acute phase. It is also important to remind that, as described before, not all neurological complications associated with dengue are caused by the direct virus neuroinvasion. Thus, some cases won't present viral RNA in CSF. Hence, the absence of viral RNA detectable in CSF do not exclude the diagnosis of neurological manifestation due to dengue infection. In a previous study, detection of viral RNA (multiplex RT PCR) and quantification of viral load (real time RT-PCR) in CSF and serum samples from dengue patients with neurological manifestation were evaluated. Discordant results were found in seven patients. Three individuals with dengue RNA in the CSF had negative serum samples obtained on the same day as the CSF and four individuals presented viral RNA in serum but not in CSF. These findings emphasize the importance of CSF analysis. Moreover, three individuals with positive result in multiplex RT-PCR in CSF did not present RNA amplification in real time RT-PCR. In contrast, the viral load was determined in two patients with negative multiplex RT-PCR. More studies are needed before the implementation of the detection and determination of viral load in CSF in routine laboratory ${ }^{2}$.

Table 1. CSF findings in different neurological manifestation associated with Dengue infection.

\begin{tabular}{lll} 
CSF analysis & & Neurological manifestation \\
\hline Inflammatory CSF: Pleocytosis, hyperproteinorhachia, blood-CSF barrier & Encephalitis & Neuromyelitis optica \\
dysfunction (albumin quotient $\left.\geq 8 \times 10^{-3}\right)$, and intrathecal synthesis of total & Myelitis & ADEM \\
$\operatorname{lgG}(\operatorname{lgG}$ index $\geq 0.7$ or oligoclonal IgG bands) & Meningitis & Polyneuropathy \\
Protein-cytological dissociation & Guillain Barré & Mononeuropathy \\
& Miller Fisher syndrome & Cerebro-meningeal hemorrhage \\
Hemorrhagic CSF & Myelitis \\
Intrathecal synthesis of dengue antibodies & Several neurological manifestation \\
Detection of dengue IgM antibodies & Encephalitis \\
Detection of viral antigens and/or viral RNA & Myelitis \\
& Meningitis & \\
\hline
\end{tabular}

*: indicates inflammatory reaction in CNS; **: indicates viral neuroinvasion. 
As the virus is an arbovirus transmitted by Aedes mosquitoes, some environmental and vector characteristics are important to the disease emergence. Several factors contribute for the increase of density and geographic distribution of the vector, enhancing the virus transmission. Among then, temperature, pluviosity, living conditions, poverty, social inequalities and lack of knowledge about disease prevention constitute determining factors for dengue transmission. Is important to highlight that as increases the virus circulation and transmission, increases the probability of severe clinical manifestation as nervous system involvement, mainly in areas with distinct serotypes circulation. Neurological complications in dengue infection become increasingly important as the dengue epidemics continue to occur. The clinical manifestations are diverse and results from different neuropathogenic mechanisms. To obtain a reliable diagnosis is of utmost importance to know the disease physiopathology. The development and validation of new diagnostic tests is important, especially regarding the NATs applications in CSF.

\section{References}

1. Guzmán MG, Kourí G. Dengue: an update. Lancet Infect Dis 2002; 2:33-42.

2. Domingues RB, Kuster GW, Onuki-Castro FL, et al. Involvement of the central nervous system in patients with dengue virus infection. J Neurol Sci 2008;267:36-40.

3. Soares CN, Cabral-Castro MJ, Peralta JM, et al. Meningitis determined by olygosymptomatic dengue virus type 3 infection: report of a case. J Int Infec Dis 2010;14:150-152.

4. Ramos C, Sánchez G, Pando RH, et al. Dengue virus in the brain of a fatal case of hemorrhagic dengue fever. J Neurovirol 1998;4:465-468.

5. Pancharoen C, Thisyakorn U. Neurological manifestations in dengue patients. Southeast Asian J Trop Med Public Health 2001;32: 341-345.

6. Pancharoen C, Kulwichit W, Tantawichien T, et al. Dengue infection: a global concern. J Med Assoc Thai 2002;85(Suppl):S25-S33.

7. Soares CN, Faria LC, Peralta JM, et al. Dengue infection: neurological manifestations and cerebrospinal fluid (CSF) analysis. J Neurol Sci 2006;249:19-24

8. Peter S, Malhotra N, Peter P, Sood R. Isolated Bell's palsy - an unusual presentation of dengue infection. Asian Pac J Trop Med 2013;6:82-84.

9. Puccioni-Sohler M, Orsini M, Soares CN. Dengue: a new challenge for neurology. Neurol Int 2012;4:65-70.

10. Miagostovich MP, Ramos RG, Nicol AF, et al. Retrospective study on dengue fatal cases. Clin Neuropathol 1997;16:204-208.

11. Bordignon J, Strottmann DM, Mosimann AL, et al. Dengue neurovirulence in mice: identification of molecular signatures in the E and NS3 helicase domains. J Med Virol 2007;79:1506-1517.

12. Jackson ST, Mullings A, Bennett F, et al. Dengue infection in patients presenting with neurological manifestations in a dengue endemic population. West Indian Med J 2008;57:373-376.

13. Soares CN, Puccioni-Sohler M. Dengue encephalitis: suggestion for case definition. J Neurol Sci 2011;306:165.

14. Wasay M, Channa R, Jumani M, et al. Encephalitis and myelitis associated with dengue viral infection clinical and neuroimaging features. Clin Neurol Neurosurg 2008;110:635-640.

15. Yamamoto Y, Takasaki T, Yamada K, et al. Acute disseminated encephalomyelitis following dengue fever. J Infect Chemother 2002; $8: 175$.
16. Kumar S, Subhashini P. Guillain Barré syndrome occurring in the course of dengue fever. Neurol India 2005;53:250-251.

17. Hughes RAC, Hadden RDM, Gregson NA, Smith KJ. Pathogenesis of Guillain Barré syndrome. J Neuroimmunol 1999;100:74-97.

18. Warke RV, Becerra A, Zawadzka A, et al. Efficient dengue virus (DENV) infection of human muscle satellite cells upregulates type I interferon response genes and differentially modulates $\mathrm{MHC}$ I expression on bystander and DENV-infected cells. J Gen Virol 2008;89:1605-1615.

19. Paliwal VK, Garg RK, Juyal R, et al. Acute dengue virus myositis: a report of seven patients of varying clinical severity including two cases with severe fulminant myositis. J Neurol Sci 2011;300:14-18.

20. Hommel D, Talarmin A, Deubel V, et al. Dengue encephalitis in French Guiana. Res Virol 1998;149:235-238.

21. Figueiredo LT, Owa MA, Carlucci RH, De Oliveira L. Laboratory diagnosis and symptoms of dengue, during an outbreak in the Ribeirão Preto region, SP, Brazil. Rev Inst Med Trop São Paulo 1992; 34:121-130.

22. Hu D, Di B, Ding X, et al. Kinetics of non-structural protein 1, IgM and IgG antibodies in dengue type 1 primary infection. Virology J 2011;8:47.

23. Guzmán MG, Kourí G. Dengue diagnosis, advances and challenges. Int $\mathrm{J}$ Infect Dis 2004;8:69-80.

24. De Paula SO, Fonseca BA. Dengue: a review of the laboratory tests a clinician must know to achieve a correct diagnosis. Braz J Infect Dis 2004;8:390-398.

25. Lanciotti RS, Calisher $\mathrm{CH}$, Gubler DJ, et al. Rapid detection and typing of dengue viruses from clinical samples by using reverse transcriptase-polymerase chain reaction. J Clin Microbiol 1992;30: 545-551.

26. CDC. The CDC Real Time RT-PCR Assay for Dengue Diagnosis. Avaiable at www.cdc.gov/dengue/clinicalLab/realTime.html. Acessed June 20th, 2013.

27. Solomon T, Dung NM, Vaughan DW, et al. Neurological manifestations of dengue infection. Lancet 2000;355:1053-1058.

28. Araújo FMC, Brilhante RSN, Cavalcanti LPG, et al. Detection of the dengue non-structural 1 antigen in cerebral spinal fluid samples using a commercially available enzyme-linked immunosorbent assay. $J$ Virol Methods 2011;177:128-131.

29. Puccioni-Sohler M, Soares CN, Papaiz-Alvarenga R, et al. Neurologic dengue manifestations associated with intrathecal specific immune response. Neurology 2009;73:1413-1417. 